\title{
Title: Preventing bulky cation diffusion in lead halide perovskite solar cells
}

Authors: Carlo A.R. Perini ${ }^{1}$, Esteban Rojas-Gatjens ${ }^{2}$, Magdalena Ravello ${ }^{1}$, Andrés Felipe Castro Mendez ${ }^{1}$, Juanita Hidalgo ${ }^{1}$, Yu An ${ }^{1}$, Ruipeng $\mathrm{Li}^{3}$, Carlos Silva-Acuña ${ }^{1,2,4}$, Juan-Pablo CorreaBaena $^{1 *}$

Affiliations:

${ }^{1}$ School of Materials Science and Engineering, Georgia Institute of Technology; Atlanta, Georgia, United States.

${ }^{2}$ School of Chemistry and Biochemistry, Georgia Institute of Technology; Atlanta, Georgia United States.

${ }^{3}$ National Synchrotron Light Source II (NSLS-II), Brookhaven National Laboratory; Upton, New York, United States.

${ }^{4}$ School of Physics, Georgia Institute of Technology; Atlanta, Georgia, United States

*Corresponding author. Email: jpcorrea@gatech.edu.

Abstract: The impact on device stability of the bulky cation-modified interfaces in halide perovskite solar cells is not well-understood. We demonstrate the thermal instability of the bulky cation interface layers used in some of the highest performing solar cells to date. X-ray photoelectron spectroscopy and synchrotron-based grazing incidence X-ray scattering measurements reveal significant changes under thermal stress in the chemical composition and structure at the surface of these films. The changes impact charge carrier dynamics and device operation, as shown in transient photoluminescence, excitation correlation spectroscopy, and solar cells. The type of cation used for passivation affects the extent of these changes, where long carbon chains provide more stable interfaces and thus longer durability (more than $1000 \mathrm{hrs}$ at $55^{\circ} \mathrm{C}$ ). Such findings highlight that annealing the treated interfaces before characterization is critical to enable reliable reporting of performances and to drive the selection between different cations.

One-Sentence Summary: We reveal the instability of interfaces treated with bulky cations, and propose long carbon chains to prevent diffusion and improve durability.

\section{Main Text:}

Bulky organic cations - too big to form a lead halide perovskite lattice - are widely used to reduce non-radiative recombination at interfaces in high-performance metal halide perovskite photovoltaics. Despite numerous reports of performance enhancement, only a few studies have investigated the actual structure and stability of the interface treated with bulky cations. (1-4) Bulky organic salts, generally halides, have been reported to interact with point defects at the perovskite surfaces and to introduce a tunneling junction with the charge extraction layers, decreasing non-radiative recombination. (1) Such salts have been used to enable power conversion efficiencies (PCE) up to $25.5 \%$ in solar cells based on halide perovskite films. (5) Bulky organic cations are frequently embedded at the perovskite and charge-transport-layer interface by deposition on already formed perovskite films, to create a uniform layer thin enough to avoid 
transport losses. Depending on the size, the deposition of bulky cations can lead to a reconstruction of the perovskite structure of lower structural dimensionality. (6)

While the passivation approach has been used widely, it is still unclear when the rearrangement of the interface occurs, if it is beneficial, or if it should be prevented, with different groups reporting contrasting results. $(1,4,7,8)$ The reconstruction of the treated interface during device operation, particularly in combination with thermal stress, could consequently result in PCE loss and shortened solar cell lifetimes, which have been neglected. In this contribution we look at the evolution in the chemical and structural composition of the interface layer when exposed to thermal stress, to understand its stability and impact on device performance. We combine synchrotron-based X-ray grazing incidence wide-angle X-ray scattering (GIWAXS), photoelectron spectroscopy (XPS), and scanning electron microscopy (SEM), with optical spectroscopy measurements such as transient photoluminescence (trPL) and excitation correlation spectroscopy, to understand how the evolution in chemical composition, structure, and microstructure of the interface induced by thermal stress impacts recombination dynamics in perovskite solar cells. Our findings demonstrate that the passivation molecules deposited at the interface slowly permeate the perovskite film under thermal stress, leading to increased nonradiative recombination. Solar cells show a correlation between the annealing of bulky cationtreated perovskite films and lower PCE. The same drop in PCE is found when devices are exposed to long-term stability testing under 1 Sun equivalent illumination at $55^{\circ} \mathrm{C}$, a temperature of interest for photovoltaics operation easily reached by solar panels during the day. We note that no abrupt performance decay is observed when devices are kept at room temperature during aging, which underlines the importance of testing at high temperature the solar cells comprising passivated perovskite films. The fast changes demonstrated by some of the most promising cations at the perovskite crystal surface show that these molecular surface modifiers do not provide long-term device operation and highlight the need for new passivation molecules able to passivate the defects at the perovskite surface without diffusing and reconstructing the perovskite bulk.

\section{Results and Discussion \\ Structure of the interface: XPS, SEM, GIWAXS}

30 To understand how bulky organic salts commonly used as passivation agents impact the optoelectronic properties and stability of halide perovskite films, we deposited triple cation lead mixed halide layers, of approximate composition in solution $\mathrm{Cs} 0.06 \mathrm{MA} 0.16 \mathrm{FA} 0.79 \mathrm{PbI}_{2.52} \mathrm{Br} 0.49$ (here referred to as CsMAFA, where MA is methylammonium and FA is formamidinium). We modified the top surface of these films with organic salts containing the same number of carbons and based on a phenyl group or a linear chain, such as phenethylammonium iodide (PEAI), octylammonium iodide (OAI), and octylammonium bromide $(\mathrm{OABr})$, which have been used for defect passivation in high-performance perovskite optoelectronics. $(7,9-11)$ The passivated samples were then thermally annealed at $100{ }^{\circ} \mathrm{C}$ for different time durations $(0,10,40 \mathrm{~min})$. A schematics of the experiment is reported in Fig. 1A. The XPS elemental scans for N1s and Pb4f of the untreated film, labeled Ref, and of the perovskite treated with PEAI and OABr are presented in Fig. 1B-E. The elemental scans for OAI-treated films, as well as the surveys and elemental scans for C1s, I3d, $\mathrm{Br} 3 \mathrm{~d}, \mathrm{Cs} 3 \mathrm{~d}, \mathrm{O} 1 \mathrm{~s}$ are presented in Fig. S1,2 in the supplementary material. Table S1 summarizes the approximate surface composition of the different films as extracted by the peak fits of the elemental scans. Comparison of the N1s peak area of FA and MA in Fig. 1B for the reference film - $\mathrm{C}=\mathbf{N}$ at $400.6 \mathrm{eV}$ (FA) and $\mathrm{C}-\mathbf{N}$ at $402.4 \mathrm{eV}$ (MA) - reveals an excess of FA and a deficiency in MA at the surface with respect to the starting solution composition (Table S1). (12) Further comparison with the $\mathrm{Pb} 4 \mathrm{f} \mathbf{P b}-\mathrm{X}$ peak where $\mathrm{X}$ is a halide, at $138.5 \mathrm{eV}$, shows the presence of an 
excess of organics at the surface, in agreement with previous reports detailing the tendency of FAI to accumulate at the exposed perovskite interface (Table S1). $(13,14)$
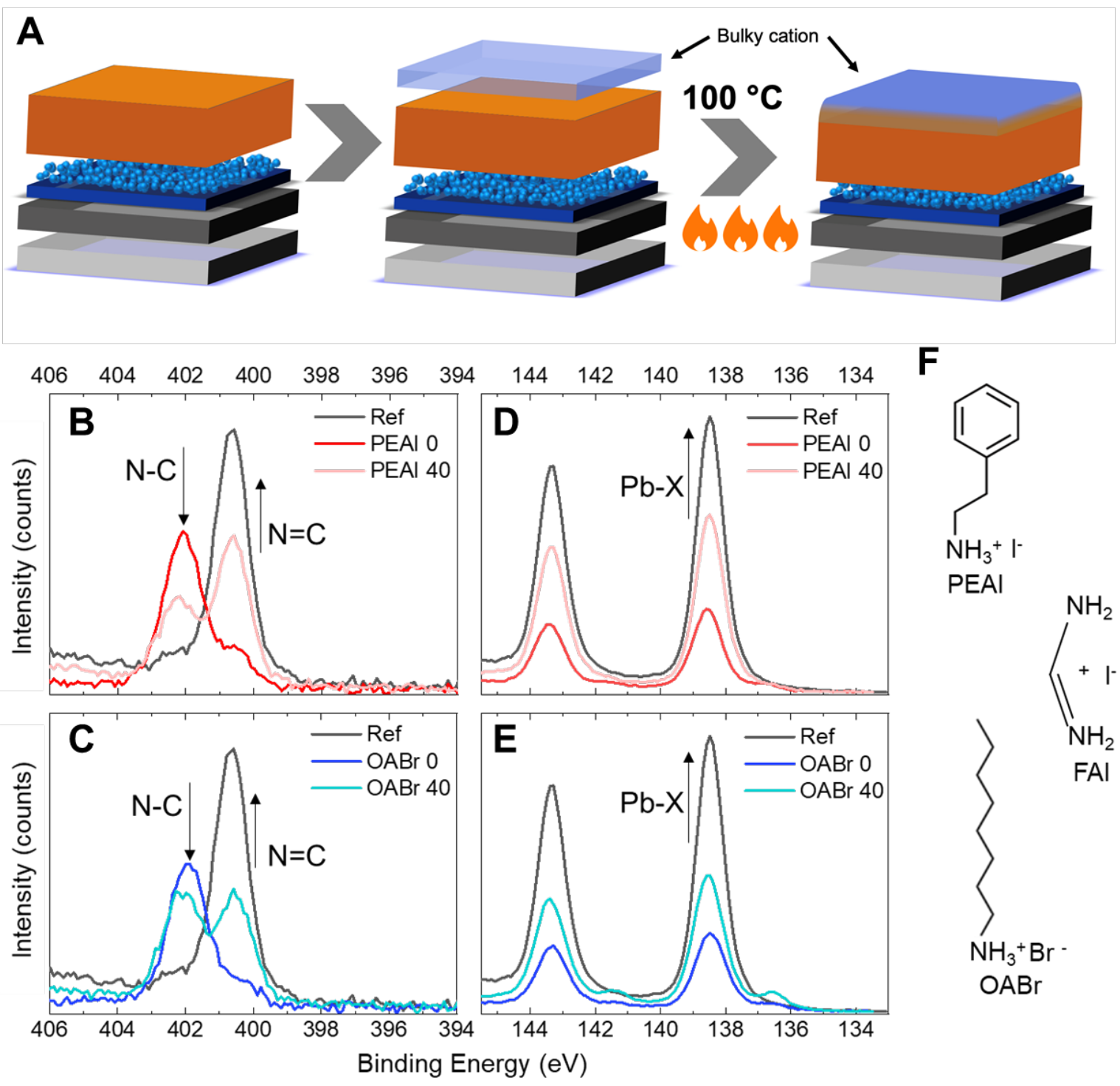

Fig. 1. Compositional evolution under thermal stress of the CsMAFA with the addition of a bulky cation layer. (A) Schematics of the experiment. XPS of N1s (B and C) and Pb4f (D and E) signals for metal halide perovskite films passivated with PEAI (B and $\mathbf{D})$ or $\mathrm{OABr}(\mathbf{C}$ and $\mathbf{E})$. The pristine CsMAFA film spectrum is reported in black. The 0 and 40 after the cation name in the legend are the annealing times in minutes, at $100{ }^{\circ} \mathrm{C}$. (F) Molecular structures of the cations shown in the XPS spectra.

The signatures of the organic cations dominate the XPS of the surface of the perovskite films treated with PEAI and OABr before annealing (labeled PEAI 0, OABr 0). The C-N peak intensity is enhanced significantly, which is attributed to the amine group of the passivant molecules, along with a reduction in the $\mathrm{C}=\mathbf{N}$ peak of FAI (Fig. 1B, D). The $\mathbf{C}-\mathrm{C} 1 \mathrm{~s}$ and $\mathbf{C}-\mathrm{N}$ 1s peaks intensity also increase with respect to the untreated perovskite film, and a broad $\pi-\pi^{*}$ satellite characteristic of aromatic compounds appears at $292 \mathrm{eV}$ in the case of PEAI (Fig. S1). (15) At the same time, the 
intensity of the $\mathbf{P b}$-X peak - used as a marker for the presence of a metal halide Ruddlesden-Popper (RP) phase at the top surface - gets suppressed, highlighting a top interface composed mostly of organic molecules (Fig. 1C, E). As for Pb, signals from FA and Cs appear strongly reduced, yet still visible, which could be ascribed to incomplete coverage of the PEAI or OABr layer or an Xray penetration depth larger than the organic layer thickness (Fig. S1). Alternatively, the $\mathrm{Pb}$ signal could be due to a RP layer beginning to form by the exchange of cations with the bottom perovskite film.

To test the stability of the interface treated with PEAI and $\mathrm{OABr}$, we annealed the films at $100{ }^{\circ} \mathrm{C}$, to favor the transition of the interface layer towards its most thermodynamically stable configuration. The XPS spectra of freshly deposited PEAI and OABr were compared with those that were annealed for $40 \mathrm{~min}$ (here referred to as PEAI 40, OABr 40, respectively). The thermal stress leads to a decrease in the $\mathrm{C}-\mathrm{N}$ peak intensity associated with the PEA and OA cations and to an increase in the $\mathrm{C}=\mathbf{N}$ FA signal (Fig. 1A, B). Concurrently, the intensity of the $\mathbf{P b}-\mathrm{X}$ signal increases, hinting at the conversion of the top interface to a RP structure or back to the original perovskite phase. The transition to this intermixed state happens relatively fast and is almost complete within the first 10 min of annealing in the case of PEAI (Fig. S2). Extending the annealing from 10 to 40 min produced only minor changes. The PEA (N-C) / Pb ratio decreases of only $10 \%$, in contrast to the $74 \%$ reduction observed within the first 10 min of annealing (Table $\mathrm{S} 1)$. For the $\mathrm{OABr}$ treated films a peak appears in the $\mathrm{Pb} 4 \mathrm{f}$ elemental scan at $136.6 \mathrm{eV}$, attributed to the formation of $\mathrm{Pb}_{0}$. The peak becomes more intense after annealing and might be related to the degradation in vacuum reported for RP phases. (16) An oxygen peak is visible in the O1s elemental scan of the CsMAFA reference $(533 \mathrm{eV}, \mathrm{C}-\mathrm{O}$ bond), which disappears in all treated films, in agreement with previous reports on the water barrier effect exerted by bulky-cation treated interfaces. $(17,18)$ To explain if the changes in surface stoichiometry observed by XPS before and after the thermal treatment are associated with microstructural changes and with the formation of an RP structure, we performed SEM and synchrotron-based GIWAXS measurements on the deposited films (Fig. 2). The introduction of the passivation layers leaves the domain size of the perovskite film almost unchanged, with grains having about $200 \mathrm{~nm}$ diameter (Fig. 2, Fig. S3). Films passivated with no post-annealing were difficult to image in the SEM, particularly when OA cations are used, possibly related to the presence of a thin amorphous/insulating organic layer on the treated surface (Fig. 2E, Fig. S3). After 40 minutes of annealing, the images become more crisp, which could be associated with the diffusion of the bulky cations and crystallization of RP phases (Fig. 2C, Fig. S3). In contrast, focus in the SEM images is difficult to achieve in the OABr film even after the annealing (Fig. 2G). GIWAXS of the films treated with PEAI shows a crystalline phase even before annealing, with diffraction at $q=0.28,0.56,0.84,1.13 \AA^{-1}$ (Fig. 2B, Fig. S4, Fig. S5), which is absent in the bare CsMAFA film (Fig. 2J). We attribute those peaks to a RP phase of the type $(\mathrm{PEA})_{2}(\mathrm{~A})_{\mathrm{n}-1} \mathrm{~Pb}_{\mathrm{n}} \mathrm{I}_{3 \mathrm{n}+1}$ with $\mathrm{n}=2$, where A could be Cs, FA, MA.(19, 20) All peak maxima belonging to the $(00 \mathrm{~h})$ family of planes $(\mathrm{h}=1,2,3,4)$ are distributed along $q_{z}$ with regular spacing, indicative of diffraction planes oriented parallel to the sample surface, which is normally the case for RP metal halides. Annealing of the PEAI treated films leads to a structural rearrangement of the interface. Within the first $10 \mathrm{~min}$ at $100{ }^{\circ} \mathrm{C}$ we observe a spread in the orientation of the RP phase without loss in the overall peak intensity (Fig. S4). As the annealing time increases to 40 min, loss in crystallinity of the $\mathrm{n}=2$ phase is observed. At the same time, new peaks at larger $q$ values of $0.30,0.61,0.93 \AA^{-1}$ increase in intensity, being visible already in the 10 min film diffraction (Fig. 2D, Fig. S4, Fig. S5). The shift of the peak positions towards larger $q$ is indicative of a reduction in the interplanar spacing, however, the shift appears too small to be attributed to a pure transition to a Ruddlesden-Popper phase with $\mathrm{n}=1$, and too large to be merely attributed to 
a change in halide or A site composition of the $n=2$ phase. (20-23) Based on our XPS results showing an increased amount of FA at the surface we hypothesize that the new peaks might be generated by partial substitution of PEA with FA in (PEA) $)_{2}(\mathrm{~A})_{1} \mathrm{~Pb}_{2} \mathrm{I}_{7}$.
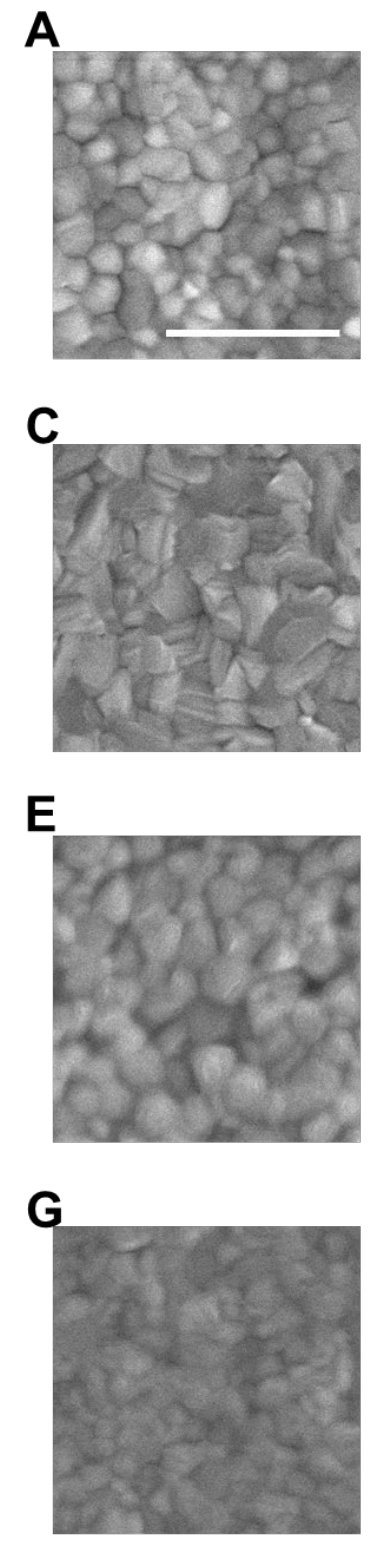

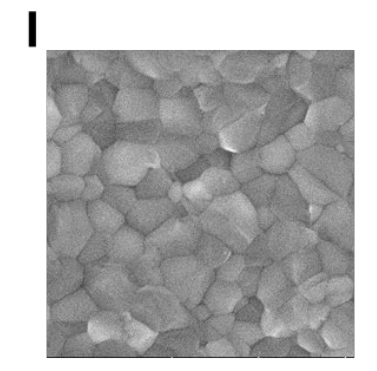

K
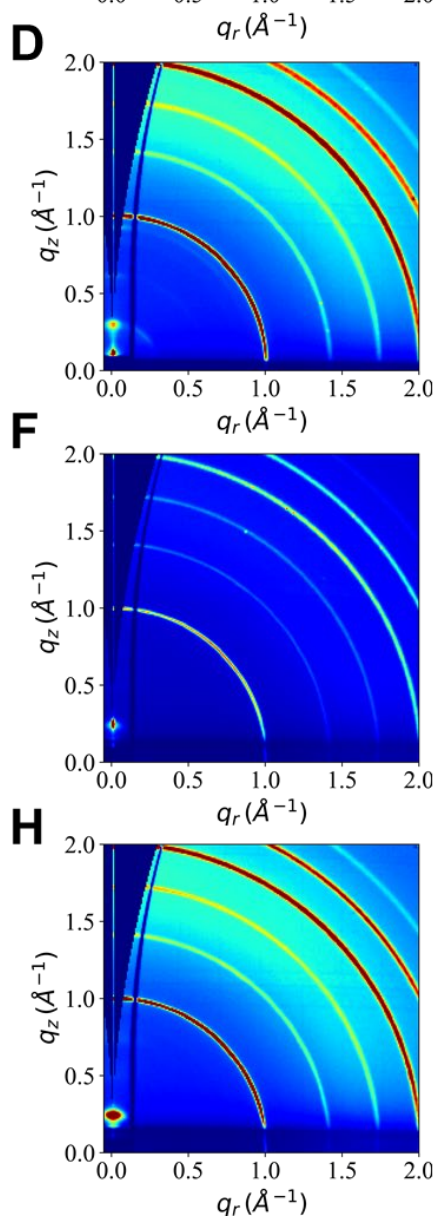

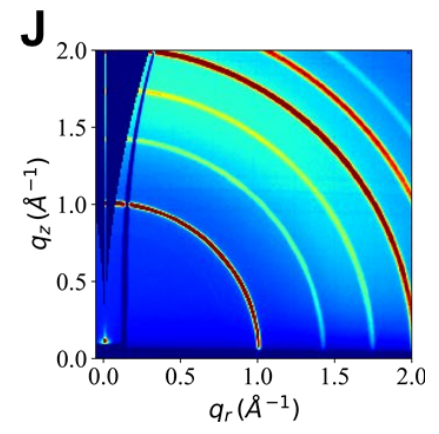

i)
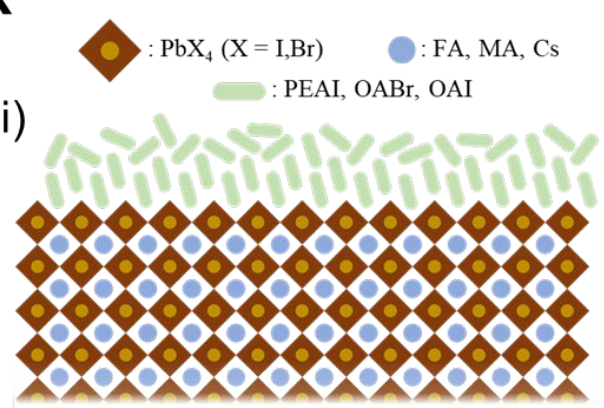

ii)

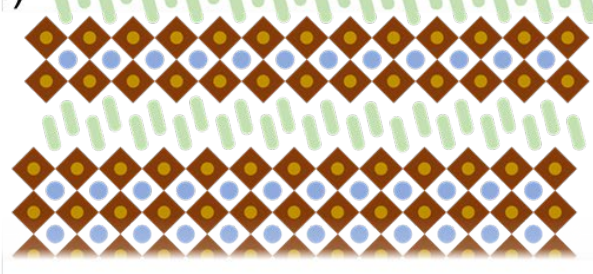

iii)

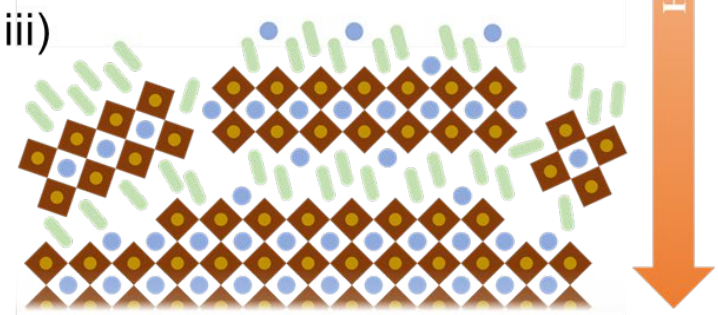

Fig. 2. Microstructure and crystal structure evolution of bulky-cation treated interfaces. SEM and GIWAXS images of: PEAI treated perovskite films before $(\mathbf{A}, \mathbf{B})$ and after $40 \mathrm{~min}$ annealing $(\mathbf{C}, \mathbf{D})$; OABr treated films before $(\mathbf{E}, \mathbf{F})$ and after annealing $(\mathbf{G}, \mathbf{H})$; reference CsMAFA film $(\mathbf{I}, \mathbf{J})$. Scale bar for the SEM images is $1 \mu \mathrm{m}$. (K) Schematics of the evolution of the interface treated with bulky-cations upon annealing.

In OA treated films no crystalline features are visible before applying thermal stress, supporting the hypothesis of the amorphous nature of the treated interface before annealing (Fig. 2F). After annealing for $40 \mathrm{~min}$ at $100{ }^{\circ} \mathrm{C}$ a weak signal appears in both $\mathrm{OABr}$ and OAI treated films, with planes at $q=0.25,0.49,0.73 \AA^{-1}$ oriented along $q_{z}$ (Fig. 2H, Fig. S4, Fig. S5). Such diffractions 
can be all related to the formation of a $n=2$ Ruddlesden-Popper phase analogously to the case of PEAI. $(24,25)$ These diffractions are more intense for the OAI-treated film than the OABr-treated samples (Fig. 2H, Fig. S4, Fig. S5). For the films treated with OA no secondary phase formation is observed, with the exception of an OABr film showing low intensity peaks and at $q$ of 0.52 and $0.78 \AA^{-1}$ in the sector average around $q_{z}(-20<\chi<20)$, which suggest an evolution towards structures with smaller interplanar spacing also for this cation, but over significant longer timescales than PEAI (Fig. S5). We note that the RP crystallinity in un-annealed films can vary from batch to batch, but evolves towards the same quasi-steady-state after $40 \mathrm{~min}$ of annealing. We observed both increases and decreases in the intensity of the RP diffraction signal within the first $10 \mathrm{~min}$ at $100{ }^{\circ} \mathrm{C}$ (Fig. S4, Fig. S6). Such variability underlines the highly unstable nature of interfaces treated with bulky cations before annealing and the importance of heating the substrates after the deposition of the organic salts to stabilize these structures.

Combining the XPS, SEM, and GIWAXS findings we can identify (1) a correlation between the type of bulky cation used and the stability of the treated interface, (2) evolution stages of the treated interface independent from the type of cation used. Three main transformation steps can be identified, which are depicted in Fig. 2K. (i) The formation of an organic overlayer: the bulky cations form an overlayer on top of the perovskite bulk, and only the very interface undergoes conversion. (ii) Bulky cation diffusion and RP phase formation: as heat is provided, the cations diffuse in the perovskite layer, inducing interface reconstruction into RP phases. This step proceeds at room temperature for PEAI. (iii) RP reconstruction and crystallinity loss: after the diffusion step, the composition of the interface layer stabilizes but the structural change continues. New low dimensional phases with smaller interplanar spacing are formed, and an overall loss in crystallinity is observed, which in PEAI is also accompanied by a loss of orientation. All the data acquired from the three techniques highlight higher thermal stability of the $\mathrm{OABr}$ and OAI treated interfaces with respect to PEAI.

\section{Interface-mediated optoelectronic properties}

Time-resolved photoluminescence (trPL) and excitation correlation photoluminescence (ECPL) Having understood the evolution in composition and structure of the treated interface, we investigated how the addition of bulky cations affects the stability of the optoelectronic properties of perovskite films and devices. Looking at the trPL decays of CsMAFA films treated with organic salts, before and after annealing we can correlate the physical changes of the interface with changes in charge-carrier recombination dynamics. As can be seen in Fig. 3A, B the annealing process and the solvent used for the deposition of the organics do not affect the photoluminescence (PL) dynamics in the film, with the film washed with isopropanol (IPA) and annealed even showing a slower PL decay. However, differences are observed once the bulky organic cations are deposited on the top perovskite surface (Fig. 3C, D), with annealing of the treated films resulting in faster PL decays. The change in carrier dynamics could be ascribed to either increased non-radiative recombination due to the formation of deep traps at the interface or to the extraction of the charge carriers into the newly formed RP phases. We note batch-to-batch variations in the relative difference in lifetimes between the reference CsMAFA film without passivation and the passivated films (Fig. S7). However, we observe consistent lifetime trends, with the trPL traces of films treated with the organic salts and annealed for 40 min always showing the faster radiative recombination dynamics (Fig. 3, Fig. S7, Fig. S8). This trend implies that the reconstruction of the 
perovskite interface promoted by annealing after bulky cation treatment is detrimental to the quality of the film and will cause PCE loss as is demonstrated above.

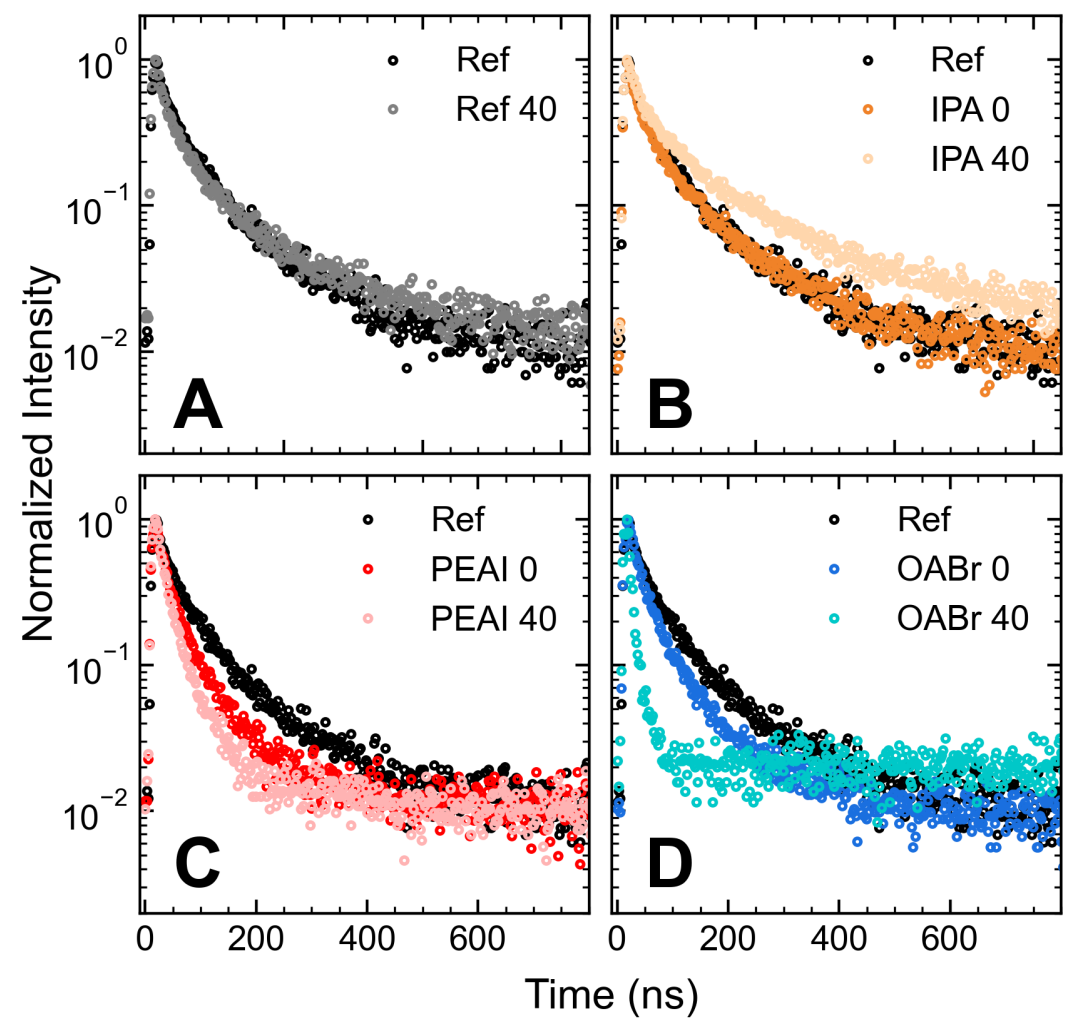

Fig. 3. Impact of bulky-cations on PL radiative lifetimes. trPL of: (A) CsMAFA reference films; (B) CsMAFA films washed with IPA to decouple the impact of bulky-cations and solvents; CsMAFA films treated with PEAI (C) or OABr (D). All films were measured before and after an annealing step at $100^{\circ} \mathrm{C}$ for $40 \mathrm{~min}$.

We further study the effect that the bulky cation treatment and annealing have on the defect population via excitation correlation photoluminescence (ECPL). We use ECPL to investigate PEAI treated films, which show the largest differences in the composition and structure of the interface before and after the annealing process at $100^{\circ} \mathrm{C}$ for $40 \mathrm{~min}$. As it has been described previously, the ECPL technique consists in exciting the sample with two pulses, each modulated at a distinct frequency $\left(\Omega_{1}, \Omega_{2}\right)$. Then, from the integrated photoluminescence the nonlinear contribution can be separated from the linear component by demodulating at the sum of the frequencies $\left(\Omega_{1}+\Omega_{2}\right)$ and at the fundamental frequencies respectively. (26-28) A time delay between the two pulses allows exploring the charge carrier population's evolution in time. The nonlinear contributions to photoluminescence, under the Shockley-Read-Hall model, are mainly caused by nonradiative recombination due to defect-assisted recombination or Auger recombination processes. $(26,27)$ Defect assisted nonradiative recombination produces a positive ECPL signal, wheres auger processes would introduce a negative ECPL component. $(26,27)$ The ECPL signals of the three films in Figure 4A-C, undergo a positive shift as the excitation density increases, which can be attributed to nonradiative recombination dominating the carrier dynamics at those excitation densities. The temporal evolution of the nonlinear carriers with and without 
interface modification and annealing is in the subnanosecond time scale for all cases, which is an indication of fast trapping processes previously assigned to shallow traps. $(26,29)$ The fast trapping shows that shallow traps are dominant at the excitation densities probed since deep traps in lead halide perovskites show slower recombination ranging from nanoseconds to microseconds and would display very slow ECPL dynamics. This does not mean that there are no deep traps in the perovskite, it implies that at the excitation density range used, the deep traps are filled and the shallow traps dominate the nonlinear recombination. Negative values of ECPL response observed at high excitation density in lead halide perovskites have been assigned to Auger processes, to the $\gamma_{\text {Auger }} n^{2} p$ term in particular, assuming the electron is the carrier being trapped. $(26,27)$ However, negative values at low excitation density $\left(10^{17} \mathrm{~cm}^{-3}\right)$ have not been fully interpreted, we hypothesize this contribution being related to the $\gamma_{\text {Auger }} n p^{2}$.
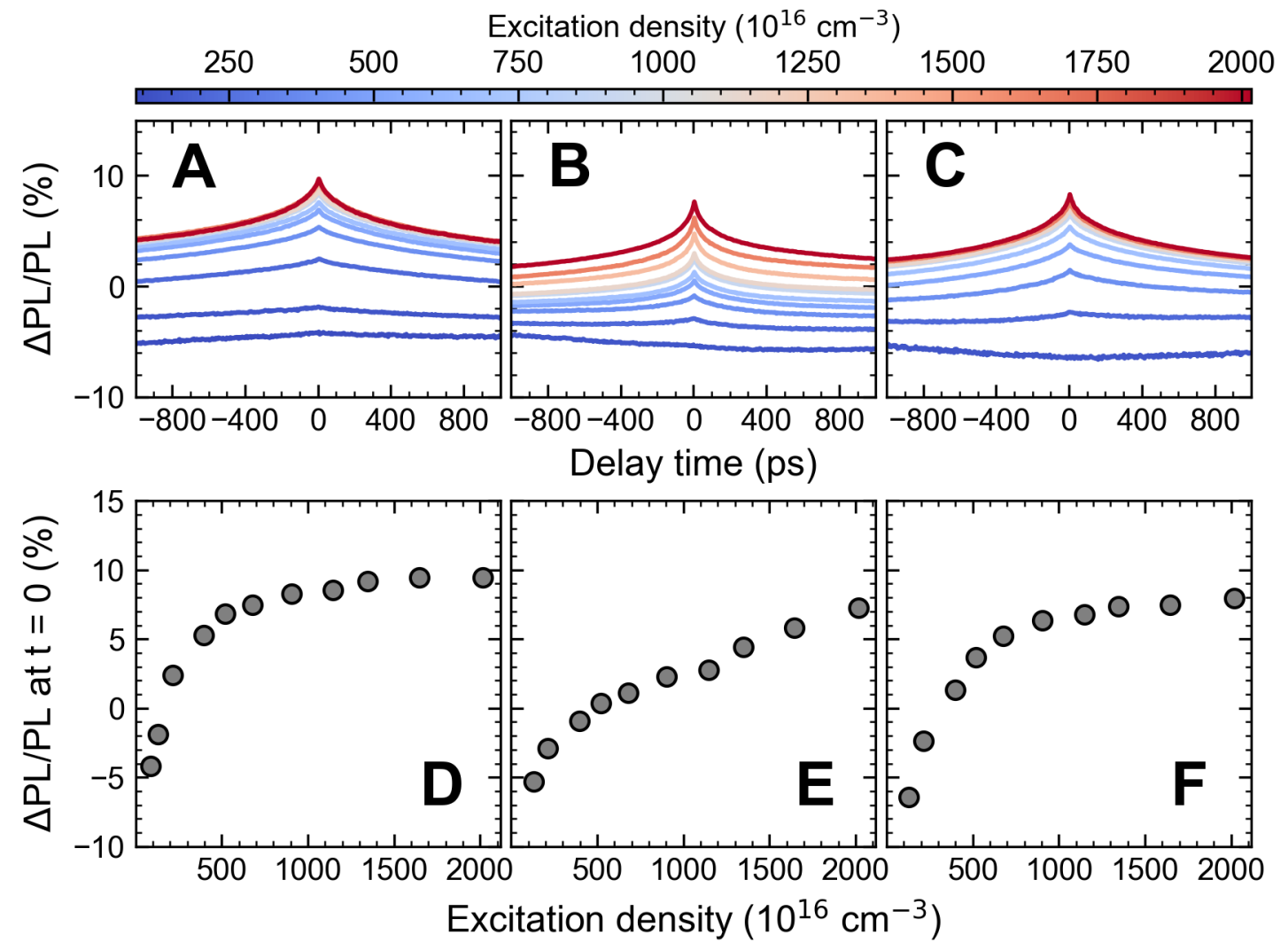

Fig 4. Effect of PEAI treatment on shallow traps density. ECPL dynamics and excitation density dependence of the ECPL at time zero of: (A, D) CsMAFA film, (B, E) CsMAFA film treated with a PEAI solution and $(\mathbf{C}, \mathbf{F})$ CsMAFA film treated with a PEAI solution annealed for $40 \mathrm{~min}$.

Comparing the ECPL response at $\mathrm{t}=0$ of the CsMAFA reference film with the PEAI solution treated film (Fig. 4D, E, respectively) it can be seen that the ECPL signal saturates at an excitation density of approximately $5 \times 10^{18} \mathrm{~cm}^{-3}$ in the CsMAFA film, while the saturation point of the PEAI treated sample is not reached in the excitation density range probed. This change in the saturation threshold implies an increase in the density of shallow traps in the film after the treatment with the PEAI solution. Although treatment with iodine has been used to passivate defects related to iodine deficiency, recent works suggest shallow defects are caused by excess iodine in the surface of lead halide perovskite films. $(11,30)$ We attribute to this latter effect the increase in shallow traps observed in our measurement, which is also in agreement with the 
increase in the surface halides $(\mathrm{I}+\mathrm{Br}) / \mathrm{Pb}$ ratio observed after the treatment with PEAI in XPS, from 4.04 to to 7.65 (Table S1). If the film treated with PEAI is further annealed, the initial scenario of shallow traps is recovered, as shown in Fig. 4F. This recovery in the ECPL response behavior is also accompanied by a decrease in the $(\mathrm{I}+\mathrm{Br}) / \mathrm{Pb}$ surface ratio, which moves from 7.65 to 5.07 . We hypothesize the decrease in halides at the surface to be associated with their incorporation in the interphase layers formed upon annealing. Note that although there is a decrease in the shallow traps caused by the thermal annealing, the trPL results indicate that annealing increases nonradiative recombination by forming deep traps which are not observed whithin the excitation density range observed in the ECPL experiment.

Device performance analysis

To understand how the evolution of the interface impacts the performance of a complete solar cell, we fabricated devices based on the architecture Glass $|\mathrm{FTO}| \mathrm{TiO}_{2}-\mathrm{c}\left|\mathrm{TiO}_{2}-\mathrm{mp}\right| \mathrm{CsMAFA}$ | spiroOMeTAD | Au (Fig. 4A), where FTO is Fluorine-doped Tin Oxide, $\mathrm{TiO}_{2}$-c a compact layer of titanium dioxide, $\mathrm{TiO}_{2}$-mp a thin film of $\mathrm{TiO} 2$ nanoparticles, spiro-OMeTAD 2,2',7,7'tetrakis(N,N-di-p-methoxyphenyl-amine)9,9'-spirobifluorene. We focused on the study of the PEAI and $\mathrm{OABr}$ molecules only, given the similar behavior of $\mathrm{OABr}$ and OAI salts. When included, the organics were deposited on top of the CsMAFA layer, before spiro-OMeTAD. The results reported here are from devices measured three days after deposition of the Au electrode, as all solar cells - the CsMAFA non-passivated reference in particular - show enhancements in opencircuit voltage $\left(V_{o c}\right)$ and short-circuit current $\left(J_{s c}\right)$ within this timeframe (Fig. S9). The reverse current-voltage scans $(J-V)$ of the champion devices for each variation are reported in Fig. S10. The distributions of $V_{o c}, J_{s c}$, and stabilized PCE of the devices are reported in Fig. 4B-D. The medians and standard deviations of the photovoltaic parameters are summarized in Table 1. As can be seen in Fig. 4D, the introduction of a bulky organic salt at the CsMAFA interface leads to a small increase in the average stabilized PCE if the films are not exposed to thermal stress, mostly benefiting from a reduced hysteresis with respect to the reference. The PCEs decrease when the bulky-cation layers are annealed, with the largest drop suffered by the devices that include PEAI. The decrease in PCE is induced by a simultaneous loss in the median $V_{o c}$, fill factor $(F F)$, and $J_{s c}$ values when the organic-treated films are annealed (Fig. 4B, C, Fig. S9B). We hypothesize two different processes inducing such losses: (i) an increased thickness of the passivation layer induced by the diffusion of the organic cation and by the conversion of the perovskite interface to low dimensional phases; (ii) the formation of electronic traps at the treated interface as a consequence of annealing. Process (ii) in particular could originate the simultaneous drop in the $V_{o c}, F F, J_{s c}$ of annealed devices, whereas an increase in series resistance (i) would only impact $F F$ and $J_{s c}$. Both effects appear to be present in PEAI devices, as evidenced by the increase in series resistance in dark $J-V$ scans (Fig. S13) and by the trPL trends shown in Fig. 3. Both (i) and (ii) keep progressing after the first $10 \mathrm{~min}$ of annealing (Fig. S8, Fig. S12), raising concerns on the long-term operational stability of 
A

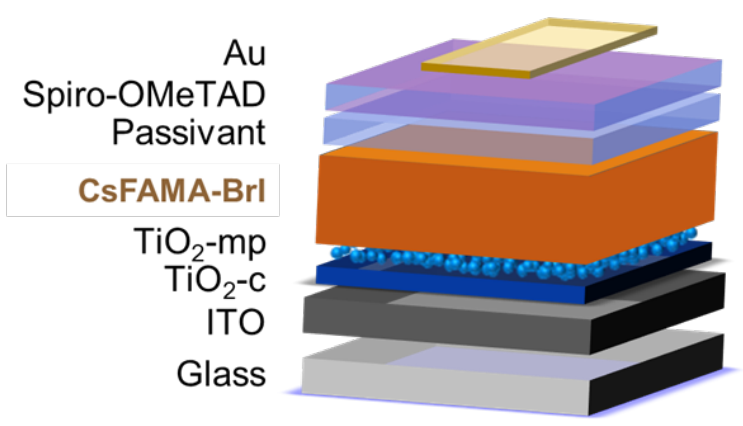

C

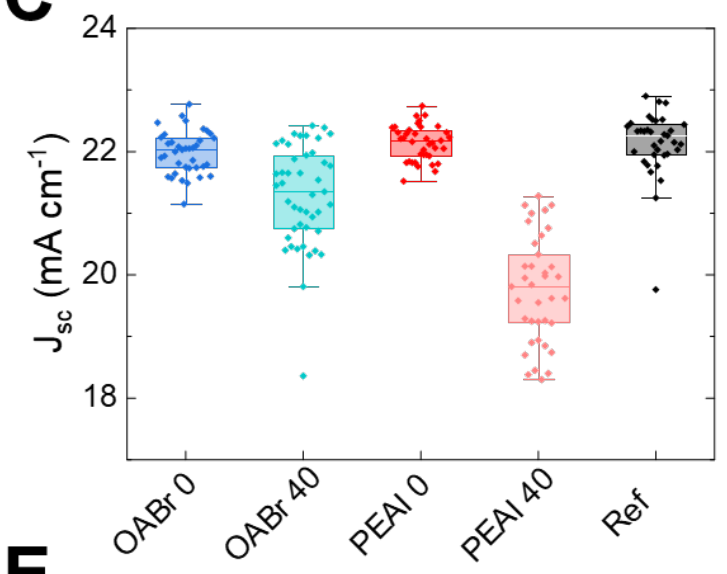

B

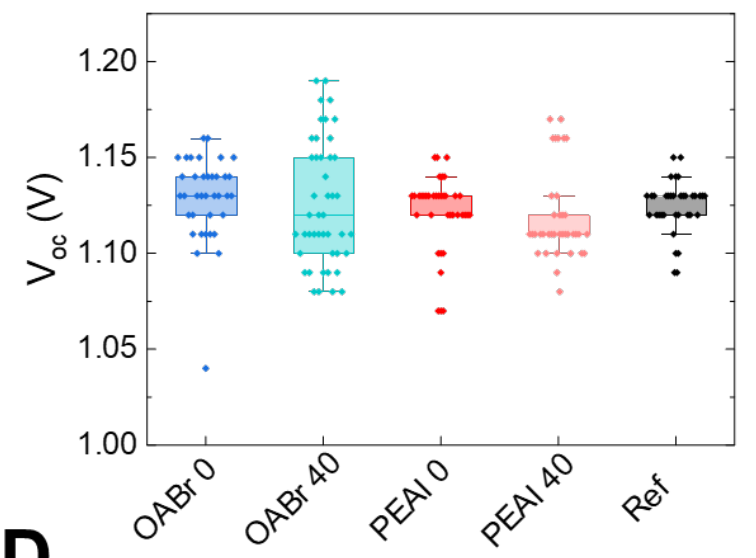

D

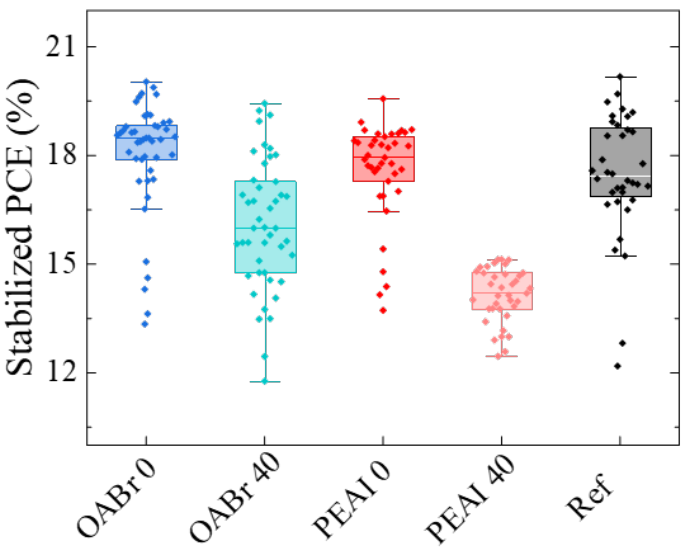

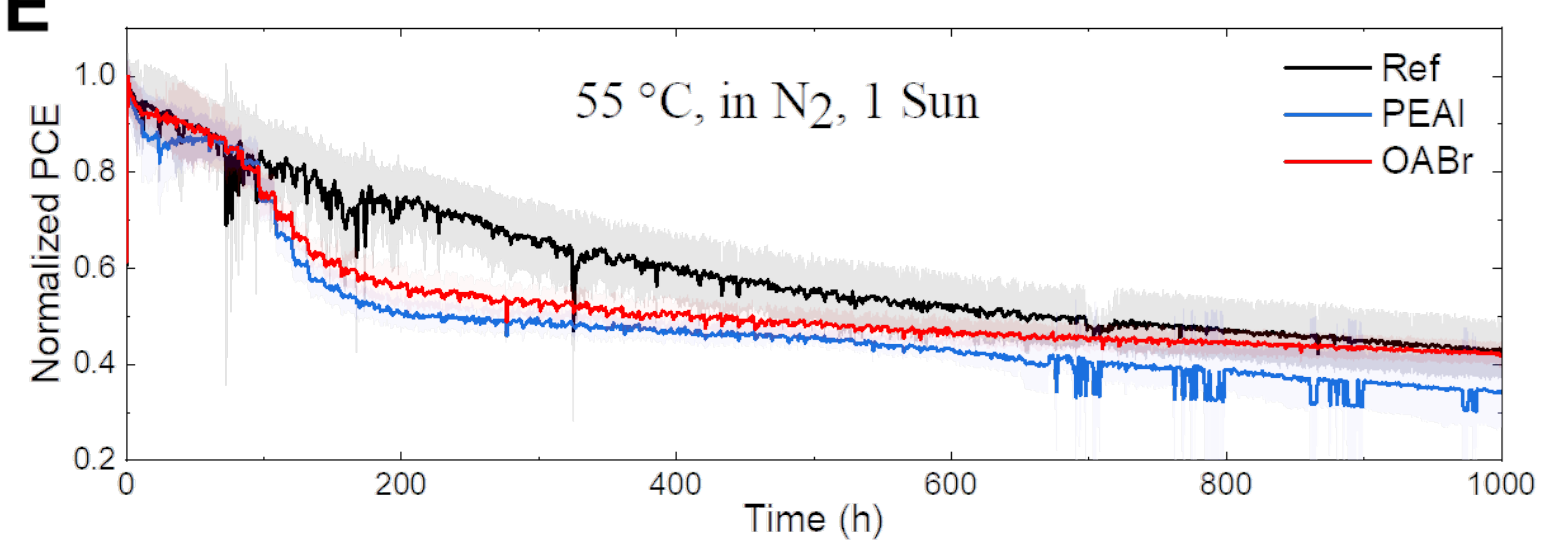

Fig. 5. Solar cell structure, photovoltaics parameters, stability. (A) Solar cell stack used in this work. (B), (C) $V_{\text {oc }}$ and $J_{\text {sc }}$ distributions as extracted from the reverse J-V scan. (D) PCE value after maximum power point tracking for $>1 \mathrm{~min}$. More than 36 independent solar cells were fabricated for each variable to produce the boxplots in (B-D). (E) Maximum power point tracking under equivalent 1 sun illumination in $\mathrm{N}_{2}$ atmosphere, $55^{\circ} \mathrm{C}$ of the untreated CsMAFA film versus the layer treated with PEAI or OABr without any further annealing.

perovskite devices treated with these cations. In $\mathrm{OABr}$ treated films no evident contribution from the series resistance is visible (Fig. S13), suggesting a performance decay mostly dominated by 
(ii). The $V_{o c}$ drop observed in both films could be induced by the excess halide introduced with the deposition of the organic salts, resulting in the formation of $I_{i}$-mediated trap centers. (11) As can be seen in Fig. 4B, the highest $V_{o c}$ s are reached by films including organic cations and annealed for $40 \mathrm{~min}$, despite the drop in the median $V_{o c}$ values (Table 1), reaching a maximum of $1.19 \mathrm{~V}$ for the $\mathrm{OABr}$ samples and of $1.17 \mathrm{~V}$ for the PEAI, against the $1.15 \mathrm{~V}$ of the reference. This apparently contradictory result can be explained by noting that a $V_{o c}$ enhancement is observed also in the CsMAFA reference exposed to $40 \mathrm{~min}$ extra of annealing (Fig. S9B). Disuniformities in the coverage of the organic layer might thus result in a dominance of the $V_{o c}$ enhancement effect of annealing and explain the presence of outliers.

Table 1. Photovoltaic parameters in numbers. Median and standard deviations of photovoltaic parameters extracted from $\mathrm{J}-\mathrm{V}$ scans in reverse ( $\mathrm{Rv}$, bias changing from positive to negative value) and in forward direction (Fw, bias changing from negative to positive value). The stabilized PCE distribution after $>1$ min maximum power point tracking is reported in the rightmost column. In bold the PCE of the champion devices for each variation.

\begin{tabular}{|c|c|c|c|c|c|}
\hline & $V_{o c}(V)$ & $J_{s c}\left(m A c m^{-2}\right)$ & $F F(\%)$ & PCE (\%) & Stabilized PCE (\%) \\
\hline \multirow{2}{*}{$\mathrm{OABr} 0$} & Rv $1.13( \pm 0.02)$ & $22.0( \pm 0.3)$ & $75.4( \pm 5.4)$ & $18.8( \pm 1.5)$ & \multirow{2}{*}{$18.4( \pm 1.5) \mathbf{2 0 . 0}$} \\
\hline & Fw $1.09( \pm 0.03)$ & $21.9( \pm 0.4)$ & $70.3( \pm 3.9)$ & $16.8( \pm 1.3)$ & \\
\hline \multirow{2}{*}{ OABr 40} & $\mathrm{Rv} 1.12( \pm 0.03)$ & $21.4( \pm 0.8)$ & $69.3( \pm 3.8)$ & $16.7( \pm 1.5)$ & \multirow{2}{*}{$16.0( \pm 1.8) \mathbf{1 9 . 4}$} \\
\hline & Fw $1.08( \pm 0.03)$ & $21.3( \pm 0.9)$ & $67.5( \pm 3.2)$ & $15.3( \pm 1.4)$ & \\
\hline \multirow{2}{*}{ PEAI 0} & Rv $1.13( \pm 0.02)$ & $22.2( \pm 0.3)$ & $72.9( \pm 5.2)$ & $18.1( \pm 1.4)$ & \multirow{2}{*}{$17.9( \pm 1.4) \mathbf{1 9 . 6}$} \\
\hline & Fw $1.09( \pm 0.02)$ & $22.0( \pm 0.3)$ & $67.7( \pm 3.8)$ & $16.3( \pm 1.0)$ & \\
\hline \multirow{2}{*}{ PEAI 40} & $\mathrm{Rv} 1.11( \pm 0.02)$ & $19.8( \pm 0.9)$ & $62.8( \pm 3.8)$ & $14.0( \pm 1.2)$ & \multirow{2}{*}{$14.2( \pm 0.7) 15.1$} \\
\hline & Fw $1.07( \pm 0.02)$ & $19.0( \pm 0.9)$ & $62.8( \pm 1.5)$ & $12.9( \pm 0.6)$ & \\
\hline \multirow{2}{*}{ Ref } & Rv $1.13( \pm 0.01)$ & $22.2( \pm 0.5)$ & $75.2( \pm 6.2)$ & $18.7( \pm 1.7)$ & \multirow{2}{*}{$17.4( \pm 1.7) \mathbf{2 0 . 2}$} \\
\hline & FW $1.07( \pm 0.02)$ & $22.0( \pm 0.6)$ & $65.9( \pm 5.9)$ & $15.5( \pm 1.6)$ & \\
\hline
\end{tabular}

We investigated further if the changes observed upon annealing in the structure, chemical composition, and electronic performances, of interface-modified perovskite films are also present in conditions of relevance for solar cell operation. We thus exposed the solar cells to 1 Sun 
equivalent illumination, at $55^{\circ} \mathrm{C}$ in an inert atmosphere, and used a maximum power point (MPP) tracking algorithm to monitor the decay in their PCE. Every $12 \mathrm{~h}$ we performed a $J-V$ scan to retrieve the evolution in $J_{s c}, F F, V_{o c}$ during aging. Fig. 4F presents a comparison of the stability of the untreated device versus solar cells including a PEAI or OABr layer. Fig. S14 reports the results in the photovoltaic parameters extracted from the $J-V$ scans. Fig. S15 includes a comparison of the stability of treated devices aged at $25^{\circ} \mathrm{C}$ versus $55^{\circ} \mathrm{C}$. Similar to what was observed for the annealing at $100{ }^{\circ} \mathrm{C}$, the organic-treated perovskite cells aged at $55^{\circ} \mathrm{C}$ show decays in PCE faster than the reference untreated layer, mediated by simultaneous drops in $V_{o c}, J_{s c}$, and $F F$ (Fig. S14). While the decay in $V_{o c}$ observed in this instance might be related to the IPA treatment of the surface, the reductions in $J_{s c}$ and $F F$ are solely related to the introduction of the bulky cations (Fig. S14). Importantly, the decay of the photovoltaic parameters significantly slows after the first 200 $\mathrm{h}$ of light exposure, suggesting the possibility to stabilize the device interfaces with an initial annealing step. If interface-treated devices are kept at $25^{\circ} \mathrm{C}$ during the stability measurement no drop in performance is observed. Dynamics of relevance for photovoltaic operation might thus be screened if the stability testing is performed at room temperature. Such observations stress the need for a more thorough analysis of the dynamics of bulky-cation interface layers under thermal stress, and the need to test devices at temperatures $\geq 55^{\circ} \mathrm{C}$ to ensure reporting performances and stability relevant for photovoltaic operation.

\section{References and Notes}

1. C. A. R. Perini, T. A. S. Doherty, S. D. Stranks, J.-P. Correa-Baena, R. L. Z. Hoye, Pressing Challenges in Halide Perovksite Photovoltaics - From the Atomic to Module Level. Joule. 5, 1-7 (2021).

2. L. Chouhan, S. Ghimire, C. Subrahmanyam, T. Miyasaka, V. Biju, Synthesis, optoelectronic properties and applications of halide perovskites. Chem. Soc. Rev. 49, 2869-2885 (2020).

3. A. A. Sutanto, R. Szostak, N. Drigo, V. I. E. Queloz, P. E. Marchezi, J. C. Germino, H. C. N. Tolentino, M. K. Nazeeruddin, A. F. Nogueira, G. Grancini, In Situ Analysis Reveals the Role of 2D Perovskite in Preventing Thermal-Induced Degradation in 2D/3D Perovskite Interfaces. Nano Lett. 20, 3992-3998 (2020).

4. G. Yang, Z. Ren, K. Liu, M. Qin, W. Deng, H. Zhang, H. Wang, J. Liang, F. Ye, Q. Liang, H. Yin, Y. Chen, Y. Zhuang, S. Li, B. Gao, J. Wang, T. Shi, X. Wang, X. Lu, H. $\mathrm{Wu}$, Stable and low-photovoltage-loss perovskite solar cells by multifunctional passivation. Nat. Photonics, doi:10.1038/s41566-021-00829-4.

5. Best Research-Cell Efficiency Chart | Photovoltaic Research | NREL, (available at https://www.nrel.gov/pv/cell-efficiency.html).

6. G. W. Kim, A. Petrozza, Defect Tolerance and Intolerance in Metal-Halide Perovskites. Adv. Energy Mater. 10, 1-6 (2020).

7. Q. Jiang, Y. Zhao, X. Zhang, X. Yang, Y. Chen, Z. Chu, Q. Ye, X. Li, Z. Yin, J. You, Surface passivation of perovskite film for efficient solar cells. Nat. Photonics (2019), doi:10.1038/s41566-019-0398-2.

8. J. J. Yoo, G. Seo, M. R. Chua, T. G. Park, Y. Lu, F. Rotermund, Y.-K. Kim, C. S. Moon, N. J. Jeon, J.-P. Correa-Baena, V. Bulović, S. S. Shin, M. G. Bawendi, J. Seo, Efficient perovskite solar cells via improved carrier management. Nature. 590, 587-593 (2021). 
9. L. Fu, H. Li, L. Wang, R. Yin, B. Li, L. Yin, Defect passivation strategies in perovskites for an enhanced photovoltaic performance. Energy Environ. Sci. 13, 4017-4056 (2020).

10. H. Kim, S. U. Lee, D. Y. Lee, M. J. Paik, H. Na, J. Lee, S. Il Seok, Optimal Interfacial Engineering with Different Length of Alkylammonium Halide for Efficient and Stable Perovskite Solar Cells. Adv. Energy Mater. 9 (2019), doi:10.1002/aenm.201902740.

11. S. Tan, I. Yavuz, M. H. Weber, T. Huang, C. H. Chen, R. Wang, H. C. Wang, J. H. Ko, S. Nuryyeva, J. Xue, Y. Zhao, K. H. Wei, J. W. Lee, Y. Yang, Shallow Iodine Defects Accelerate the Degradation of $\alpha$-Phase Formamidinium Perovskite. Joule. 4, 2426-2442 (2020).

12. Q. Zhou, L. Liang, J. Hu, B. Cao, L. Yang, T. Wu, X. Li, B. Zhang, P. Gao, HighPerformance Perovskite Solar Cells with Enhanced Environmental Stability Based on a (p-FC 6 H 4 C 2 H 4 NH 3 ) 2 [PbI 4 ] Capping Layer. Adv. Energy Mater. 9 (2019), doi:10.1002/aenm.201802595.

13. B. Philippe, M. Saliba, J. P. Correa-Baena, U. B. Cappel, S. H. Turren-Cruz, M. Grätzel, A. Hagfeldt, H. Rensmo, Chemical Distribution of Multiple Cation ( $\mathrm{Rb}+, \mathrm{Cs}+, \mathrm{MA}+$, and FA+) Perovskite Materials by Photoelectron Spectroscopy. Chem. Mater. 29, 3589-3596 (2017).

14. J. D. McGettrick, K. Hooper, A. Pockett, J. Baker, J. Troughton, M. Carnie, T. Watson, Sources of $\mathrm{Pb}(0)$ artefacts during XPS analysis of lead halide perovskites. Mater. Lett. 251, 98-101 (2019).

15. M.-C. Tang, S. Zhang, T. J. Magnanelli, N. V. Nguyen, E. J. Heilweil, T. D. Anthopoulos, C. A. Hacker, Unraveling the compositional heterogeneity and carrier dynamics of alkali cation doped 3D/2D perovskites with improved stability. Mater. Adv. 2, 1253-1262 (2021).

16. Y. J. Hofstetter, I. García-Benito, F. Paulus, S. Orlandi, G. Grancini, Y. Vaynzof, Vacuum-Induced Degradation of 2D Perovskites. Front. Chem. 8, 1-10 (2020).

17. S. W. Knipe, J. R. Mycroft, A. R. Pratt, H. W. Nesbitt, G. M. Bancroff, X-ray photoelectron spectroscopic study of water adsorption on iron sulphide minerals. Geochim. Cosmochim. Acta. 59, 1079-1090 (1995).

18. H. Zhu, Y. Liu, F. T. Eickemeyer, L. Pan, D. Ren, M. A. Ruiz-Preciado, B. Carlsen, B. Yang, X. Dong, Z. Wang, H. Liu, S. Wang, S. M. Zakeeruddin, A. Hagfeldt, M. I. Dar, X. Li, M. Grätzel, Tailored Amphiphilic Molecular Mitigators for Stable Perovskite Solar Cells with 23.5\% Efficiency. Adv. Mater. 32, 1907757 (2020).

19. D. J. Morrow, M. P. Hautzinger, D. P. Lafayette, J. M. Scheeler, L. Dang, M. Leng, D. D. Kohler, A. M. Wheaton, Y. Fu, I. A. Guzei, J. Tang, S. Jin, J. C. Wright, Disentangling Second Harmonic Generation from Multiphoton Photoluminescence in Halide Perovskites using Multidimensional Harmonic Generation. J. Phys. Chem. Lett. 11, 6551-6559 (2020).

20. M. E. F. Bouduban, V. I. E. Queloz, V. M. Caselli, K. T. Cho, A. R. Kirmani, S. Paek, C. Roldan-Carmona, L. J. Richter, J. E. Moser, T. J. Savenije, M. K. Nazeeruddin, G. Grancini, Crystal Orientation Drives the Interface Physics at Two/Three-Dimensional Hybrid Perovskites. J. Phys. Chem. Lett. 10, 5713-5720 (2019). 
21. Y. Liu, Y. Zhang, Z. Yang, H. Ye, J. Feng, Z. Xu, X. Zhang, R. Munir, J. Liu, P. Zuo, Q. Li, M. Hu, L. Meng, K. Wang, D. M. Smilgies, G. Zhao, H. Xu, Z. Yang, A. Amassian, J. Li, K. Zhao, S. F. Liu, Multi-inch single-crystalline perovskite membrane for highdetectivity flexible photosensors. Nat. Commun. 9, 1-11 (2018).

22. M. E. Kamminga, H. H. Fang, M. R. Filip, F. Giustino, J. Baas, G. R. Blake, M. A. Loi, T. T. M. Palstra, Confinement Effects in Low-Dimensional Lead Iodide Perovskite Hybrids. Chem. Mater. 28, 4554-4562 (2016).

23. D. Ma, Y. Fu, L. Dang, J. Zhai, I. A. Guzei, S. Jin, Single-crystal microplates of twodimensional organic-inorganic lead halide layered perovskites for optoelectronics. Nano Res. 10, 2117-2129 (2017).

24. A. Lemmerer, D. G. Billing, Synthesis, characterization and phase transitions of the inorganic-organic layered perovskite-type hybrids [( $\mathrm{C} \mathrm{nH} \mathrm{2n+1NH} \mathrm{3)} 2 \mathrm{PbI} 4], \mathrm{n}=7,8,9$ and 10. Dalt. Trans. 41, 1146-1157 (2012).

25. Y. Hua, D. Hong, S. Wan, Y. Lei, M. Xie, W. Yang, Y. Du, Y. Tian, Ethanol induced structure reorganization of 2D layered perovskites (OA)2(MA)n-1PbnI3n+1. J. Lumin. 220 (2020), doi:10.1016/j.jlumin.2019.116981.

26. A. R. Srimath Kandada, S. Neutzner, V. D’Innocenzo, F. Tassone, M. Gandini, Q. A. Akkerman, M. Prato, L. Manna, A. Petrozza, G. Lanzani, Nonlinear Carrier Interactions in Lead Halide Perovskites and the Role of Defects. J. Am. Chem. Soc. 138, 13604-13611 (2016).

27. D. A. Valverde-Chávez, E. Rojas-Gatjens, J. Williamson, S. Jariwala, Y. Shi, D. P. McCarthy, S. Barlow, S. R. Marder, D. S. Ginger, C. Silva-Acuña, Nonlinear photocarrier dynamics and the role of shallow traps in mixed-halide mixed-cation hybrid perovskites. J. Mater. Chem. C. 9, 8204-8212 (2021).

28. D. Rosen, A. G. Doukas, Y. Budansky, A. Katz, R. R. Alfano, Time resolved luminescence of photoexcited p-type gallium arsenide by population mixing. Appl. Phys. Lett. 39, 935-937 (1981).

Acknowledgments: Carlo A.R. Perini thanks Monikandan Rebhadevi for the useful discussion and study material provided for the analysis of the XPS data collected in this work. Esteban Rojas-Gatjens thanks. David A. Valverde-Chavez and Jacob Williamson for their participation in the construction of the ECPL experiment, and Ajay Ram Srimath Kandada for a fruitful discussion regarding the ECPL interpretation.

\section{Funding:}

U.S. Department of Energy's Office of Energy Efficiency and Renewable Energy (EERE) under the Solar Energy Technologies Office Award Number DE-EE0008747. Recipients (CSA and ERG). U.S. Department of Energy's Office of Energy Efficiency and Renewable Energy (EERE) under the Solar Energy Technologies Office Award Number DEEE0009369. Recipients (CP and JPCB). 


\section{Author contributions:}

Conceptualization: CARP, JPCB

Data Curation: CARP, MR, ERG, RL

Formal Analysis: CARP, ERG, RL

Investigation: CARP, MR, AFCM, JH, YA, ERG

Visualization: CARP, JPCB, ERG

Funding acquisition: JPCB, CSA

Project administration: CARP, JPCB, CSA

Software: CARP, ERG

Supervision: JPCB, CSA

Writing - original draft: CARP

Writing - review \& editing: CARP, JPCB, ERG, AFCM, JH, YA

Competing interests: Authors declare that they have no competing interests.

Data and materials availability: All data are available in the main text or the supplementary materials.

\section{Supplementary Materials}

Materials and Methods

Figs. S1 to S16

Tables S1 